\section{Major histocompatibility complex}

Histocompatibility. By George D. Snell, Jean Dausset and Stanley Nathenson. Pp. xiv +401 . (Academic: New York, San Francisco and London, 1976.) \$29.50; $£ 18$.

THIS is an excellent book for seasoned professionals and advanced students with a substantial background in mammalian genetics and immunology. Emphasis is on the much studied, major histocompatibility complex (MHC) of mice $(H-2)$ and men $(H L A)$. It is difficult to find fault with this masterfully written treatise. The coverage is thorough and well-documented. The subject index is admirably detailed. The list of abbreviations given at the outset is helpful in coping with inevitable acronyms. Because so much of the current literature deals with the findings of the past few years, it is also refreshing to encounter each theme in historical perspective. One only wishes the scope extended to non-mammalian vertebrates and invertebrates, but this is newer territory awaiting exploration in depth. This superbly comprehensive volume will be the definitive reference for histocompatibility characteristics of mice and men for years to come. If progress continues at the present rate, however, an updated edition will surely be desired about 1980. The principles so nicely explained in this book should of course remain valid, but the details will surely become more complex.

The first eight chapters take the reader progressively from essential principles of transplantation immunogenetics through allogenetic polymorphism, $H-2$ complex loci, and immune response ( $I r$ ) genes in mice. In chapters 9,10 and 12 , the serology, genetics, and disease associations of the HLA complex in man are described in a cogent manner. Chapter 11 deals comparatively with the biochemistry of $H-2$ and $H L A$ alloantigens. The predominant role played by the MHC in cell interactions, especially lymphocyte interactions such as mixed lymphocyte reactions, cell-mediated lysis, T-B lymphocyte collaboration, and hybrid resistance in mice is admirably presented, especially in chapter 8 .

At any given time, every field has a prevailing dogma and histocompatibility or transplanatation immunogenetics is no exception. Although emphasis is on the MHC as the only gene complex imposing a "very strong barrier to transplants", the roles of other $H$ - or Ir genes are trenchantly considered in chapters 3 , 7 , and 13 , the final chapter. Even recipients of $H L A$-identical organ transplants will usually reject such allografts promptly in the absence of drug immunosup- pression; immunodeficient recipients of allogenic bone marrow regularly succumb to fatal graft-versus-host disease despite identity for all four $H L A$ loci. Other $H$ systems then may often have 'major' impact. Moreover, as the authors aver in several chapters, slight preimmunisation with donor-type alloantigens may convert a weak non-MHC barrier into a strong one. Uniqueness of the MHC, if indeed each species has only one such main system (book italics), apparently resides in the contiguity of the constituent loci and their interaction. Many of the constituent $\mathrm{H}-2$ loci taken singly in tests between recombinant congenic lines have effects similar to moderate non- $H-2$ loci. In light of other recent evidence, much of the mammalian genome may be organised into large functional units with relevant developmental and regulatory information associated with component structural genes.

Snell, Dausset and Nathenson deal perceptively with discordant findings. For example, divergent data concerning the genetic 'laws' of transplantation, sources of specific immune blocking, and the chemistry of MHC antigens are evaluated in a commendably evenhanded manner. Moreover, they suggest further experiments to resolve ambiguities. An understanding of sequential HLA gene-antigen relationships in man has now become difficult for non-specialists. An illustrative diagram proceeding from the chromo- some No. 6 loci $\rightarrow$ alleles and antigens $\rightarrow$ haplotypes $\rightarrow>$ phenotype would have been useful in chapter 9 to epitomise the immunogenetics of the HLA system.

Chapter 7 deals concisely with immune response genes and probable mechanisms of $I r$ gene action in mice. This theme is pursued again in man in an incisive chapter 12 on histocompatibility genes and disease-especially associations bebetween HLA and disease susceptibilities. The authors favour the hypothesis that Ir and metabolic genes closely linked to the $H L A$ genes are actually responsible for susceptibility or resistance. Although acknowledging the undoubted relevance of the $H L A$ complex to medical genetics, the reader should retain reservations toward the 'MHC controls everything' viewpoint by remembering that many known Ir genes are not linked to the $\mathrm{MHC}$; that only a few human diseases (all nonneoplastic) reveal a strong correlation with particular HLA specificities; and that animals below the phylogenetic level of advanced amphibians or bony fishes cope quite well immunologically without having evolved a mammaliantype $\mathrm{MHC}$. Altogether, this volume is an outstanding contribution to the field of transplantation genetics and immunobiology. W. H. Hildemann

W. H. Hildemann is Professor of Immunogenetics and Dental Research Institute Director at the University of California, Los Angeles, California.

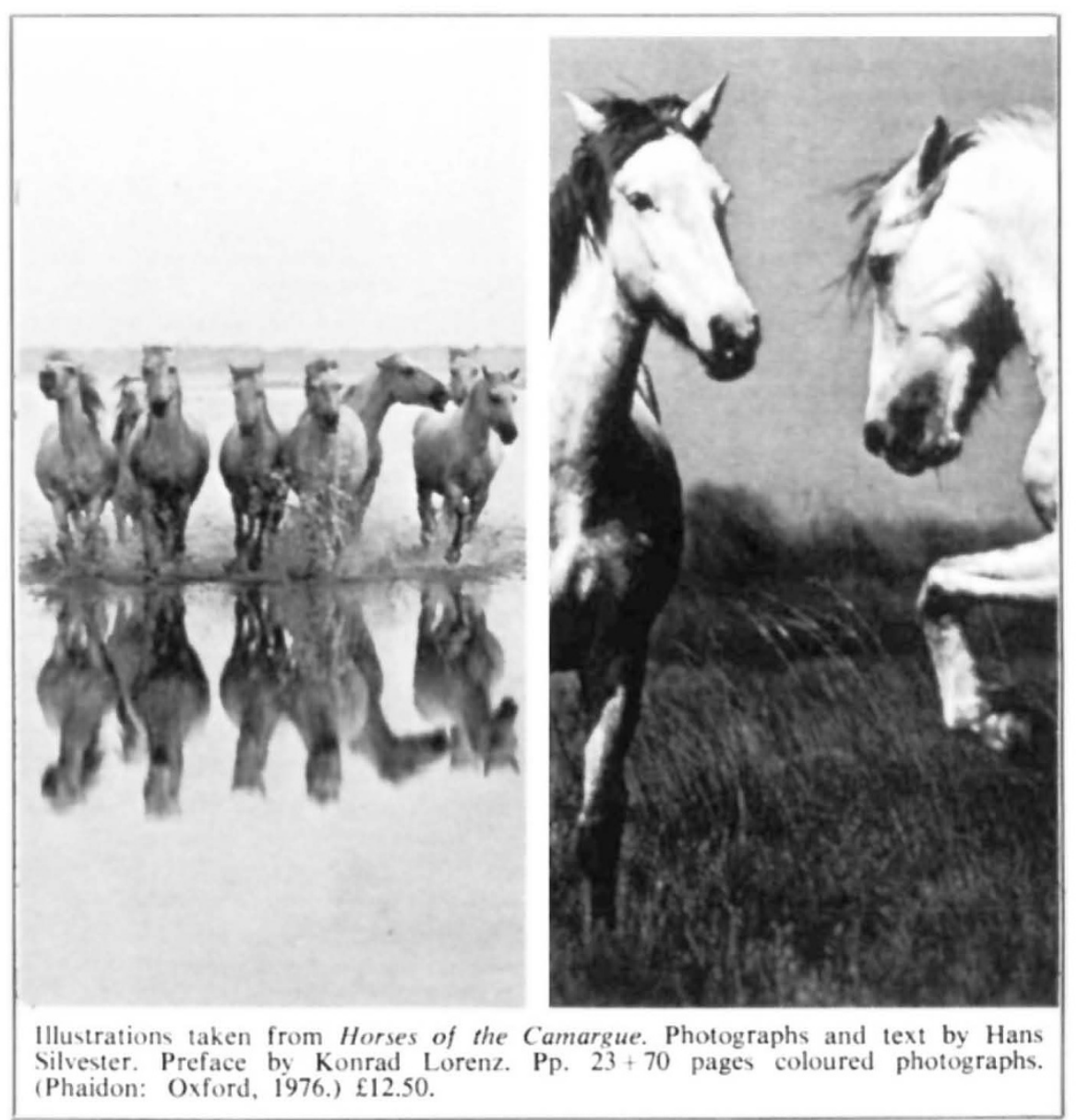

